\title{
The Research in Mobile Learning Based on Android Smartphone Platform Application
}

\author{
Di Jiaqi, Wang Jianhua, Zhang Long \\ computer college \\ China harbin normal university \\ di_jacky@163.com,wjh@vip.163.com, zlwalkman@sina.com
}

\begin{abstract}
Mobile learning is a relatively new research area, it is the basis of digital learning by the effective combination of mobile computing technology, make learners can at any time at any place you want to get the knowledge information, realizing the real sense of autonomous learning, finally realize the socialization and lifelong learning.

The Android operating system based on Linux is launched nearly 1-2 years of technical platforms, but because the Linux based operating system is a set of Android open-source mobile phone operating system of standardization, strong commonality and Internet sex, and does not exist any previous hinder the exclusive mobile industry innovation barriers, so to get a cell phone manufacturers and operators, relies on its popular in the flexibility of the system, openness, and open Internet concept, will benefit us under the information technology environment, realize liberalization, individuation, multiple mobile learning have a positive impact.
\end{abstract}

Keywords-Android, Mobile Learning, Smartphone, Learning platform

As mobile computing and wireless communication technology development, the mobile learning (M - learning) as digital learning [E - a branch of learning] are increasingly attention and become education technology and related fields of new research hotspot. Mobile learning as a kind of brand-new learning in school education and training form of field application of immeasurable potential, but so far, both theoretical research and application practice, mobile learning is still at the primary stage. How to make achievements of development and application of the new technologies, building more efficient practical mobile learning system, excavate the potential application of mobile learning, is worth deeply research. Through the Android Smartphone platform for the development of meet the needs of the user mobile learning system provides new ideas. Mobile phone users to get rich content selective, and the online teaching resources between teachers and students can through instant messaging and E-mail, in addition to two-way communication by transfer graphical user information to create regional position of similar mobile study group.

\section{MOBILE LEARNING}

Mobile learning is refers to the use of wireless mobile communication network technology, with wireless mobile communication equipment [such as mobile phones and personal digital assistant PDA, Pocket PC, etc] get education information, education resources and education services of a new digital learning form. [1] At present, the mobile learning in foreign research mainly focuses on in Europe and North America partial economy developed countries. From the research purpose to points basically has two kinds, one kind is learning by current E - launched, more for enterprise providers of training; Another kind is initiated by education institutions, used to improve teaching and learning and management.

For example in Helsinki, Finland, the university of UniWap mobile learning project, in the project by training teachers are equipped with a WAP phones, they once had and learning theme related ideas, can immediately by WAP phones will view sent to teaching server. So, the other members of the team by training in the cases not by WAP phones always read through these views.

China mobile learning research started late, low level, smaller scale, and mainly is carried out under the ministry of education planning, focusing on the construct campus LAN and based on short message mobile education research. For example, the ministry of education " Mobile Education " project. The project uses China Mobile's GPRS platform to provide SMS services to teachers and students, while teachers and students can enjoy more preferential mobile phone business, to establish a " Mobile Education "service station system for participation in "Mobile Education" project provides a variety of Services. Although the traditional education model has made great progress, but no true sense of mobile learning, and it exists problems, such as learning system instability exists, operational poor, resource-poor course of study, learning time, flexibility and interaction Poor.

\section{ANDROID SMARTPHONE PLATFORM}

A. Based on the Android platform software system structure.

Android phone is a standard Smartphone. The so-called Smartphone, refers to "the same as personal computers, with separate operating system, installed by the user software, games and other third-party service provider process, through such programs to continue to expand the functionality of the phone, and can be achieved through the mobile communication network to access the wireless network, so that the general term for a class of mobile phone."[2] 
Android is designed specifically for mobile terminal software platform and its software architecture from the bottom is divided into four levels, as shown in Figure 1:

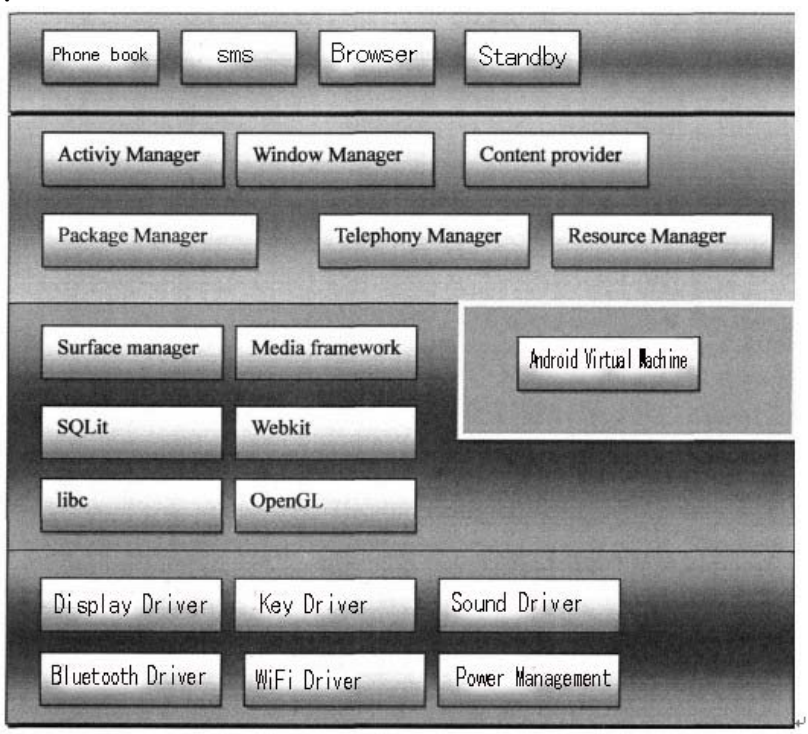

Figure 1. Android software system structure

(1)The core parts: the main system management, resource scheduling and provides support to various peripheral devices.

(2)Libraries and Android Run Time

(3) Application Framework: the main provider of API interface for the upper application call, write a standalone application.

(4)Application: such as phone book, SMS, call records, alarm clock, calculator, call of applications, primarily written with Java syntax.

\section{B. The advantage of Android operating system.}

The operating system is the basis of Smartphone platform, which provides process scheduling, task manager, file systems, network protocols and the phone peripherals such as keyboards, LCD, Buletooth, WiFi and other equipment management. The Choice of operating systems has a direct relationship between product stability, cost, and development prospects.

The mainstream of the current Smartphone market, the operating system are the windows Mobile, symbian, IPhone, Android.

Windows Mobile is a famous Microsoft designed for intelligent Mobile terminal development in the operating system, Due to the Windows operating system used for, the user easy to get started, and compatible PC swing many file format. The defect is that the system is relatively high share of resources, easy to collapse, and Microsoft needs to develop products commission, while the operating system's user interface is relatively obscure, bad user experience.

Symbian is currently the largest market share of Smartphone operating system, mainly due to the amount of NOKIA large market share of products, NOKIA symbian
Smartphone operating system mainly used. The operating system applies to the operation with the keyboard. The defect is that the mainstream media format support is poor; development requires authorization; With the prevalence of large-screen touch keyboard, the system is still in the keyboard operation mode of operation.

Apple's IPhone leads the current trend of Smartphones: cool visual effects, convenient user has and the faster speed of response. But unfortunately not opening to the outside world is a private system.

Android system achieved the completely open to the user, anyone, any units can download the free code for development and design of mobile devices with its own mobile terminal. At the same time, this system of Ul design also accords with current trends. Motorola, Samsung, LG, Sony Ericsson and other large manufacturers have followed suit, using the system to develop their own intelligence products. Based on Android development, no matter from the technical (developers have the system all the code) or from the development costs on the (no royalties), we will not be controlled by others, it has been more and more manufacturers and developers welcome. The following are the salient features of Android system:

1) Openness.

Android, such as Linux, completely free of user-developed, the user can use their own needs secondary development of the system to meet the various needs of different areas. Manufacturers in the development process also eliminates the previously required the development of Smartphone platform licensing fees, lower development costs, and promote the development of Smartphones.

2) Google's appeal and boost.

Google as a world famous search engine giant has the strong technical strength and power to promote the development of the system.

3) All the famous manufacturer to join.

Motol, Qualcomm, TI, HTC, China Mobile and other world-renowned chip manufacturers, manufacturers, operators added to accelerate the development of the system.

4) A mature Linux system

5) Strong portability and powerful operation

\section{ANDROID SMARTPHONE PLATFORM FOR MOBILE LEARNING SUPPORT}

This stage, the mobile phone-based mobile learning in China has a wide range of applications; first let us see a group of authoritative statistical data: 
A. March 29, 2011 China Unicom released in 2010 reported earnings, that 2010 the company mobile users totaled 1.674 billion, up $13.4 \%$.

B. March 30, 2011 China Telecom Group announced in Beijing that ended at 10:58 on 29 March 2011 the number of China telecom TianYi mobile subscribers break 100 million.

C. Mobile phone users: 1-2 months, the cumulative net increase of mobile phone users nationwide 19.826 million, reaching 8.78829 million. In the mobile phone users, $3 G$ net increase of 8.938 million users to reach 55.989 million.

China's 3G network deployment began in 2009, and in the first quarter of 2011 increased by 135 million users . This makes the $3 \mathrm{G}$ in China is expected to exceed 2010 growth rate of Internet users, and the growth rate in China last year were increased.347 million 3G mobile phone users.

Analysts said China's Smartphone sales to promote the growth of $3 \mathrm{G}$ subscribers. According to analyses international, In 2009, China only sold 21 million Smartphones, and this figure increased in 2010 almost tripled to 62 million. China this year is expected in the Smartphone sales will reach 95 million departments.

According to China Internet Network Information Center, outside the data, as of the end of 2010, China had 303 million mobile Internet users, 230 million more than last year.

The above data shows: China has the world's largest mobile phone user groups, the national mobile phones have far more than the number of computers. Our country has nearly 3 million people often use mobile phone access data, including 95 million people used to use Smartphones retrieving data information. And this number is still growing at double rate. How to make the 95 million people through networking phone also can obtain knowledge, accept education, and how to let their phone to obtain knowledge, accepted education, is a time urgent and important research subject. Mobile learning be vividly portrayed. Thus, this paper studies the realization of mobile learning terminal platform technology, have broad application prospects, can create enormous economic and social benefits.

\section{BASED ON THE MOBILE SMARTPHONE STUDY}

A Smartphone is the same as the computer can download and install software to develop the basic functions of mobile phones.

Norwegian University of Technology, Jan-Kristian Markiewicz developed a system running on Windows Mobile with content aware capabilities of foreign language learning system PALLAS[foreign language training system for Mobile real situations redetect put markets], used to support traditional classroom foreign language teaching [3].Central China Normal University ,Wang Jue designed a system running on the Symbian mobile English learning system, and realized the word memory, English articles etc function module. [4]
East China normal university, WangRunHua designed of a run on Windows Mobile system on Mobile learning framework, and realized the coursework, and outdoor assignments and Mobile blogs function [5].

Experimental and applied research from the above that the emergence of Smartphones, provides developers with a very malleable terminal platform, is the realization of domestic and international mobile learning technology applications and research focus and future direction.

\section{MOBILE LEARNING TERMINAL PLATFORM DESIGN}

The design of the mobile learning terminal platform integrates the current advanced mobile communication technology. Firstly, it is a networked terminal, accessing to the Internet via mobile base on the server and terminal, the information between terminal and terminal transmission. Secondly it has position perception and unique sharing function, through the built-in GPS module for their own positioning information, then through instant communication information transmission between different terminals in the position information. Finally mobile terminal with their own designs in the user database can be networked environment simple data processing.

Mobile learning terminal platform is divided into four function modules: reading module, communication module, LBS module and communication record module, fig. 2 shows.

Reading module exists alone, communication module and LBS module need to invoke the user's information in the address list. In this terminal platform, a new mobile above application framework design study shown in figure 3 . Through this frame, learners with the help of the mobile computing devices can get help in any time, any place of study. Mobile learning by use of mobile computing devices can effectively present study content and provide teachers and learners two-way communications. In addition, it also has position perception and sharing mechanism, which previous moving learning applications don't have.

China's 3G mobile communication network has been officially put into commercial trial operation; the proposed framework for mobile learning application is based on the 3G mobile communication networks with high reliability and high data transfer rate above their design. The vast majority of mobile terminal platform learning applications need to establish a wireless connection to the Internet, individual subsystem also need a special satellite receiver chip to get data, so the application framework designed to reflect the mobile network, the Internet, "Heaven" Network (satellite) " three nets " One trend.

According to the characteristics of mobile development of system underlying data format, network communication, embedded database, some of the key code given platform upon completion of this learning-based Android mobile terminal platform based on the design and implementation, and in a simulated environment verify the availability of mobile terminal platform learning. Android is an entirely open platform for third-party software developers for its development process have a greater degree of freedom, 
breaking the traditional mobile phones, that the software can only add a small number of fixed yoke. At the same time with Microsoft, Nokia and other manufacturers' different, Android operating system, free of charge to developers, so that the cost savings of nearly. Learning through the use of the mobile terminal platform Android, mobile phone users can access a wealth of selective summary of online teaching resources. The embedded Weskit browser engine used, with touch screen, advanced graphics and other Internet functions, the reading effect with the current Smartphone readers has improved significantly compared. Meanwhile the teachers and students can be two-way communication through instant messaging and E-mail, it can be said that a variety of mobile communication technologies into a single platform. As a result of a common transport protocol easy to implement with existing Smartphone's docking system; addition to the graphical user by passing the information to create the geographical location of mobile learning groups of similar, this feature can eliminate the sense of distance between mobile learners.

\section{VI. . CONCLUSION}

Although in the user experience, there is a gap between Android platform for mobile phones and the Iphone, Android's electronics market are not many good products, but these are not unreachable gap. Just closed the gap will do. In fact, far more than Smartphone market, tablet PCs, automotive information systems, television sets, have emerged the figure Android, and Android just begun.

\section{ACKNOWLEDGMENT}

This study is supported by the science and technology project of the Education Department of Heilongjiang Province (11541093) and Harbin Normal University (10XKYY07), and the Heilongjiang Provincial Key Laboratory of Intelligence Education and Information Engineering.

\section{REFERENCES}

[1] Chris.Haseman: Android Essentials[M].APress.( 2008)

[2] Chen yue.li zhi shu. LuoYi: JZME platform phone. Based on analysis and realize communication procedures. Computer application. Vol. 23(5) (2003), p.107 - 109

[3] Markiewiez J.Personalized and eontext sensitive foreign language training supported by mobile devices: master degree thesis. Trondheim: Norwegian University of Seienee and Teehnology. (2006).

[4] Wang Qiu: based on mobile learning of English vocabulary learning system research and design: "master's degree thesis.Wu han: (central China normal university 2007)

[5] Wang Run Hua: Mobile learning system research. (master's degree thesis. Shanghai. east China normal university 2006)

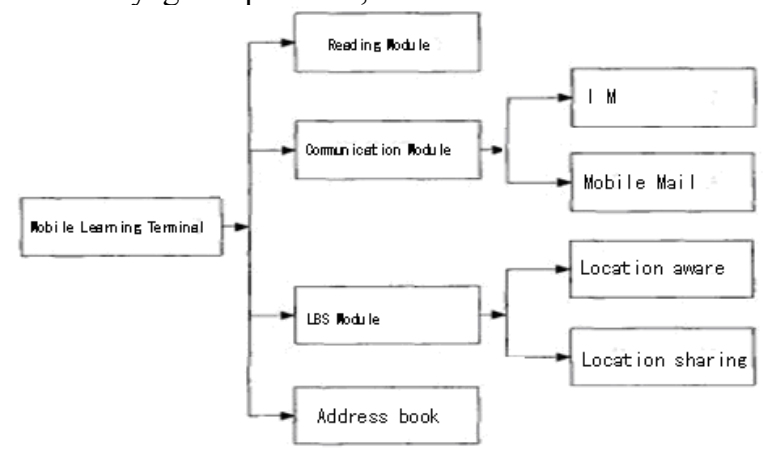

Figure 2. mobile learning terminal platform structures

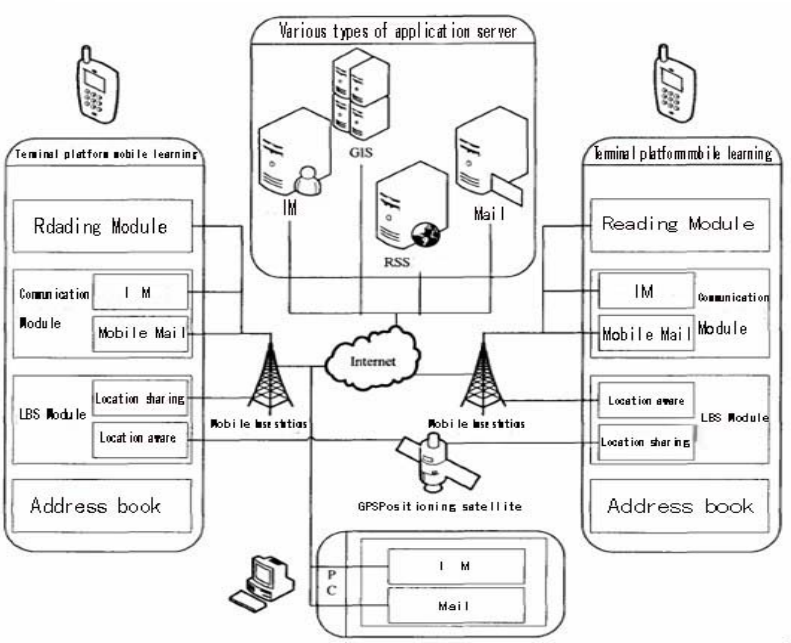

Figure 3. new mobile learning application frameworks 